\title{
The Manipulation of Algebraic Expressions: Deepening of a Widespread Difficulties and New Characterizations
}

\author{
Federica Ferretti ${ }^{1^{*}}$ \\ ${ }^{7}$ Faculty of Education, Free University of Bozen-Bolzano, ITALY \\ *CORRESPONDENCE: $₫$ federica.ferretti5@gmail.com
}

\begin{abstract}
The learning of algebra represents a difficulty within the mathematics learning process. Precisely because of their epistemological and ontological nature, semiotics provides a good key to understanding the main difficulties that hinder the learning of algebraic objects. The answers to a large scale assessments task that requires a treatment in the sense of Duval of sizeable sample of upper secondary school student is analysed. Statistical analysis allows the nationwide data to be considered according to students' ability levels. The study confirms and quantifies the distance between personal meaning and the cultural meaning attributed to algebraic objects. The difficulties of the manipulation of algebraic expressions is deepened and characterized.
\end{abstract}

Keywords: algebra, semiotics, large-scale assessments

\section{INTRODUCTION}

This paper sets out to identify and analyse the difficulties that secondary school pupils face when working with algebra, specifically focusing on issues that they may encounter in the manipulation of algebraic expressions. As highlighted by Kieran (1995, 2014), the question of algebra learning has always been a matter of interest in mathematics education research; certainly, the epistemological and ontological nature of algebraic objects attributes a crucial role to semiotic language in their learning (Duval, 1995). As this paper will reveal, it is precisely in the manipulation of various representations of algebraic expressions and the meaning attributed to them by students (Radford's Theory of knowledge Objectification - TO, 2008) that the effectiveness, or lack thereof, of algebra learning can be determined. The case study analysed involved a test administered at upper secondary school and the results refer to a sizeable sample of over 38,000 students. The research methodology adopted is a mixed method, combining quantitative and qualitative analysis to examine the situation from different points of view. The task used in the study requires the algebraic manipulation of an algebraic expression: analysis of the students' answers provides precise information regarding the difficulties encountered, and a statistical analysis using the Rasch model (Rash, 1960) allows the nationwide data to be considered according to students' ability levels as measured by the test. This study consists in the in-depth analysis and large-scale quantification of phenomena studied in the literature, as well as analysis of the phenomenon according to student ability levels. The questions that led the research are the follows: what information can be gathered regarding the manipulation of algebraic expressions and the meaning which students assign to algebraic terms, as evinced by large-scale assessment test results? With suitable statistical tools, what kind of information can we gather regarding this issue in relation with students' ability levels?

Article History: Received 18 May $2019 \bullet$ Revised 16 July $2019 \bullet$ Accepted 17 July 2019

(C) 2020 by the authors; licensee Modestum Ltd., UK. Open Access terms of the Creative Commons Attribution 4.0 International License (http://creativecommons.org/licenses/by/4.0/) apply. The license permits unrestricted use, distribution, and reproduction in any medium, on the condition that users give exact credit to the original author(s) and the source, provide a link to the Creative Commons license, and indicate if they made any changes. 


\section{Theoretical Framework}

As various studies have highlighted (Kieran, 1995; Arcavi, 1995), many students learn algebra rules by heart, with little or no conceptual understanding of their meaning. The algebraic terms, rules and properties seem to be worlds away from the thought processes of many students. Due to this situation, students then have problems tracking and applying the rules appropriately (Kieran, 2007). In particular, regarding secondary school students, Kieran observed that one of the main problems lies in the way students work with algebraic variables and expressions. The main feature of algebra learning is the transition from specific procedural knowledge to general conceptual knowledge (Sfard, 1995); this step obviously cannot exclude the attribution of meaning to algebraic terms and the manipulation of their semiotic expressions. The study carried out by Godino \& Batanero (1994) regarding understanding of mathematical terms, identifies a dialectical method between the personal understanding of the students and the institutional meaning of said terms; from the perspective of social constructivism, this occurs through practices that are held to be important in eliciting the meaning of mathematical objects (Ernest, 1991). The mathematical objects thus become cultural units which depend strongly on the context. In accordance with this line of thinking, this study embraces the approach of the TO (Radford, 2008) - in which learning is conceived as being historically and culturally constructed. Radford (2002) highlights the main features of the cognitive breakdown that students undergo when they move towards symbolic generalizations such as the process of de-subjectification and the positioning problem related to symbolic generalizations. Another factor highlighted by Radford (2002) is the scarcity of available algebraic signs; signs play a fundamental role in the learning process of algebra and this study focuses attention on referential use of signs in the management of different representations as described within Duval's approach. According to Duval, mathematical thinking and learning are identified with the coordination of semiotic systems through treatment and conversion. Treatment is the semiotic transformation from one representation to another within the same register, while conversion is the semiotic transformation of one representation to another within different registers. Duval (1993) also states that one difficulty which may emerge is due to the fact that students may identify the mathematical object with its semiotic representations (cognitive paradox); it is this possibility which leads to identifying conversion as a key cognitive function that guarantees conceptual understanding of mathematical objects, as it ensures a true distinction between the object and its representations. the most common algebraic manipulations in school practices are treatments, which is why it is difficult to establish conceptual understanding of mathematical objects. It is thus interesting to analyse what information we can gather from answers supplied in large-scale assessment projects from students tackling algebraic manipulation tasks.

\section{Methodology}

In recent years, large-scale assessment tests have played an increasingly important role in mathematics education research (De Lange, 2007). In Italy, every year since 2008, the national evaluation system institute, the INVALSI, have carried out periodic and systematic checks on students' knowledge and skills, performing large-scale Italian and Mathematics (and from this year, English) assessment tests for students from primary school to upper secondary school. The construction of the tests is the result of the work of a panel of mathematics teachers (currently about 150) and experts from different backgrounds (disciplinary, statistics, and pedagogical); the preparation process requires 21 months, during which time ex-ante qualitative analysis for construction and administration (field trial) of the pre-tests, and ex-post quantitative and qualitative analysis are carried out. The technical framework of the INVALSI mathematics test takes into account the main constructs of national and international mathematic education as well as the Italian National Guidelines (Ferretti, Lemmo, Martignone, 2018). The validation of INVALSI tests is based on the main indexes of Classical Test Theory (CTT) and use of the Rasch model (Rasch, 1960), a logistic parameter model which allows an estimated evaluation of student ability and the difficulty of each item, positing both sets of data on the same scale with values from -4 to +4 . In particular, the Rasch model expresses the probability of correct answers to a specific item according to the difficulty of the item itself and the ability of the student as measured by the entire test. The logistic curve thus produced by the model is denominated the characteristic curve. Important data on the item behaviour is provided by specific graphs, which allow a comparison between the item characteristic curve and model output with empirical data, particularly the trend of each answer option while taking into consideration the students' ability levels as demonstrated by the overall test. In this paper, the item results will be presented both in percentage terms and characteristic curve achieved by applying the Rasch model to the INVALSI sample group. As highlighted in Callingham and Bond (2006), Rasch 


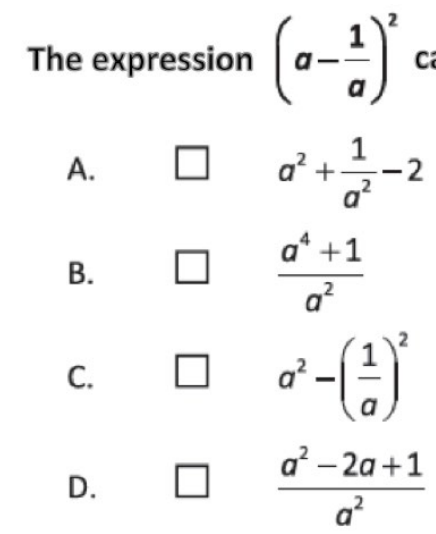

Figure 1. The task administered at nationwide in the INVALSI test to Grade 10 students in 2017

measurement is being used increasingly as a research tool; in details, the research method adopted in this study is mixed, qualitative-quantitative (Hart et al., 2009).

In detail, the research methodology intertwines the scheme: QUAN --> QUAN+QUAL, in accordance with Johnson \& Onwuegbuzie (2004). The first research stage consisted in a research among the large-scale assessment results for examples of algebraic difficulty, using the semiotic theory lens approach of Duval approach and Radford's TO. The study was carried out using the research tool GestInv, the INVALSI database already used in earlier research studies (Bolondi, Ferretti, Giberti, 2018), with which it was possible to search among all tests administered to upper secondary school students for answers to algebraic questions demonstrating the lowest national percentage of correct answers (QUAN phase). The results were used to tackle the research question of this study, an investigation of the ability to manipulate algebraic expressions, specifically the ability to perform a treatment as laid down by Duval. The last stage, the QUAN+QUAL stage consisted of combining the qualitative approach using a theoretical lens with the national results and their relative statistical analysis. In particular, the data regarding the percentage of correct answers and data regarding percentage of individual options chosen were analysed. Finally, an interpretation of the characteristic curve allowed further analysis of student performance with reference to their ability as revealed by the overall test.

\section{Results}

The task analysed in the research study was administered nationwide to all students in the second year of Italian upper secondary school. The task (Figure 1) requires the manipulation of an algebraic expression and regards content and skills expressly demanded in the Italian National Guidelines.

The task calls for an alternative way of expressing an algebraic expression; specifically, the algebraic expression in the task text is the square of a binomial. There is no context and it is not necessary to provide interpretation or modelling. The correct answer is option A; in order to select the right answer, the correct manipulation of two different algebraic representations inside the same semiotic register (a treatment, in Duval's terms) is necessary. The task was administered in a national achievement survey of approximately 545,000 upper secondary school students (precisely grade 10 students), across Italy. A sample of approximately 38,000 students was selected in order to have both geographical (of Italian regions) and typological (of study courses) representativeness.

Figure 2 shows the percentage of correct, wrong, missing and invalid answers and the individual percentages of options selected nationwide. 

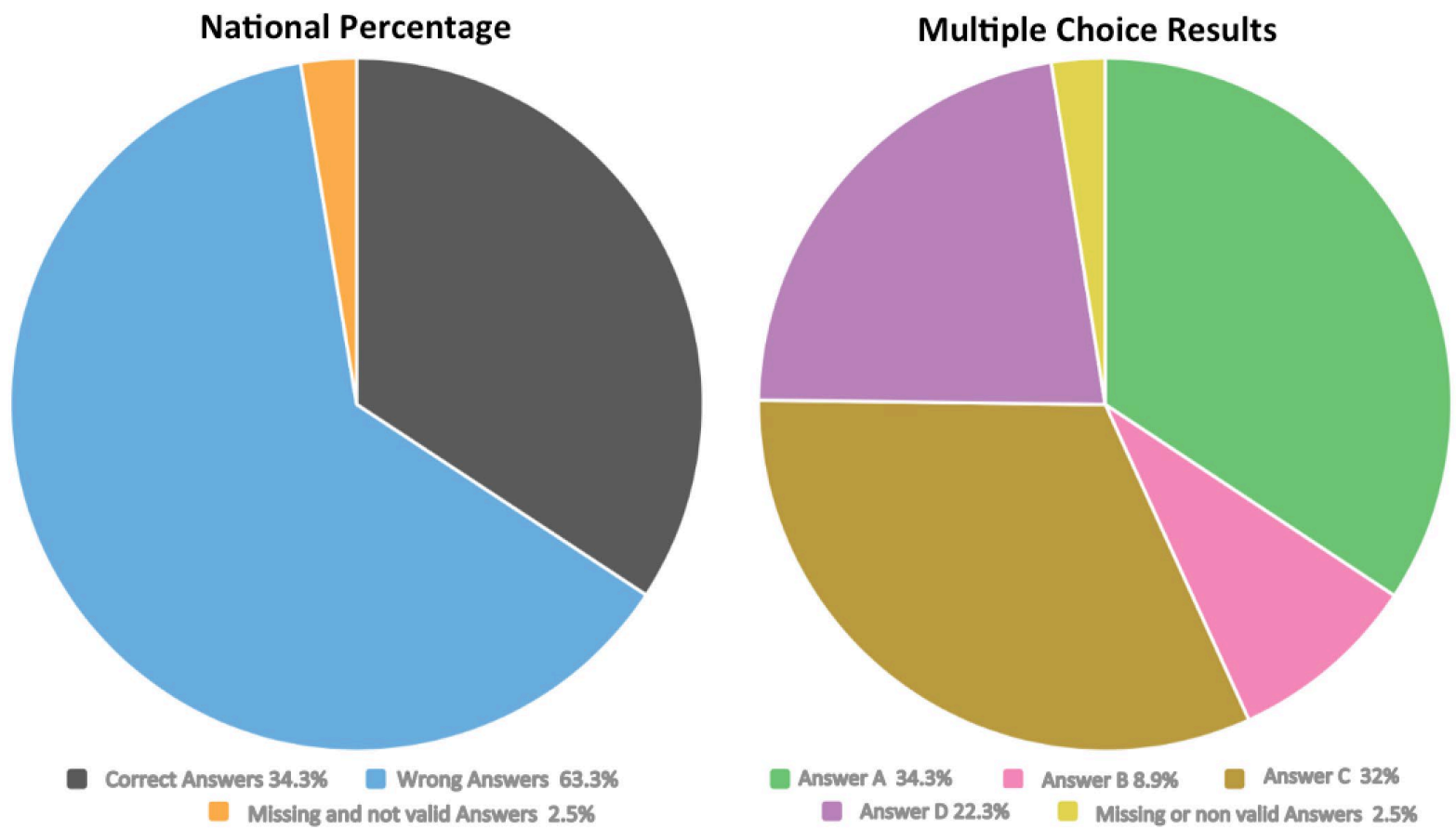

Figure 2. The percentage of answers at nationwide referring to the task investigated

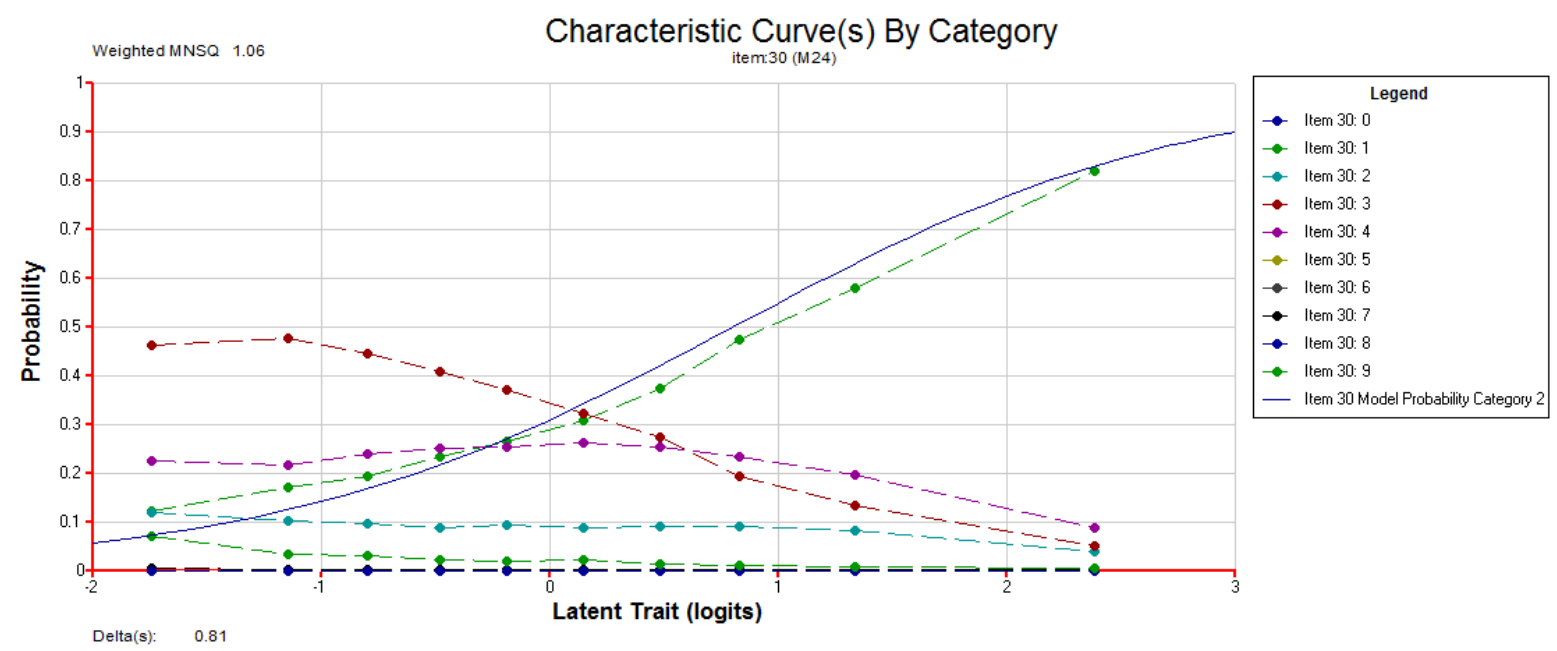

Figure 3. The percentage of answers at nationwide referring to the task investigated

As shown in the graph, the number of correct answers was lower than $35 \%$, the number of wrong answers was about $63 \%$, and the number of missing or invalid answers was very low $(2.5 \%)$. The fact that the correct answer was option A may indicate that many students did in fact read it (being the first option) but then decided to reject it. Among the wrong answers, the most popular was option $\mathrm{C}$ which involved the difference of squares of monomials from the algebraic expression in the task text.

Figure 3 displays the percentage of answers divided into deciles. The $\mathrm{x}$-axis represents student ability as estimated by test performance and the students are divided into ability groups; the $\mathrm{y}$-axis represents the probability of choice for each individual option.

As shown in the graph (Figure 3), the trend of correct answers fits quite well with the predicted statistical model (continuous blue line); this is one of the parameters which indicates that the question is good/valid as an indicator (Rash, 1960). Regarding low level abilities, the probability of choosing the correct option is only slightly higher than $10 \%$ while the probability of choosing option C (dotted red line) is almost $50 \%$. Option C remains the most popular choice up to medium-high level ability students; only from the sixth decile does the 
curve invert with a higher probability of choosing correct option A (dotted green line). In the last decile, the probability of choosing the correct option is over $80 \%$ and none of the other options exceeds $10 \%$. Another interesting fact is that as regards high ability levels (the last 3 deciles), the students who chose the wrong answer were more likely to choose option D than option C.

\section{Discussion}

In the task analysed in this study, selecting the correct answer requires a good knowledge of algebraic terms in the symbolic semiotic register. The fact that most students provide an answer (the number of missing or invalid answers is very low) indicates that the topic is familiar to students, who feel able to answer the question. In this task, the contextual meaning is not provided to the student; the algebraic manipulations required are all in the same semiotic register and so, given the very low number of correct answers, difficulty is obviously encountered due to this factor. The advantage of access to such large-scale assessment results in a closed task allows us to analyse the mistake in greater depth. In analysing the students' answers, it emerges in fact that most students who made a mistake did so by choosing option C. More than 170,000 students nationwide chose this option, with more than 12,000 doing so in the sample group. The algebraic expression used in option $\mathrm{C}$ is the square difference of monomials which make up the algebraic expression of the item text; the cultural meaning of the mathematical item is different from the personal understanding of the students who seem to mechanically perform the routine manipulation, without aligning meaning or relation to personal cultural meaning far removed from the cultural meaning of the mathematical task (Radford, 2008). Analysis of the characteristic curve supplies precise information regarding student behaviour and their mathematical ability as measured over the entire test. As the characteristic curve reveals (Figure 3), up to medium-high ability levels, students are more likely to choose the wrong answer, particularly opting for option $\mathrm{C}$ rather than the correct answer A. This indicates that the phenomenon studied here does not only affect "weaker" students but also a large number of those students who demonstrate higher ability. Finally, the fact that the performance trend for high-ability students who make mistakes consists in an inversion of the curve for options $\mathrm{C}$ and $\mathrm{D}$ offers precise information on the type of problems that different ability level students may encounter in resolving the task. In fact, the mistake that leads students to choose the algebraic expression in option D may be due more to distraction than a lack of attribution of personal meaning to the semiotic representation. This adds yet another layer of interest to the phenomenon in question. Finally, very particular is the behaviour of option $\mathrm{B}$, which seems to be independent from the ability of the students. This option corresponds to a very "naif" manipulation of algebraic symbols, and the fact might be interesting to explore as a further issue.

\section{Conclusion}

As various studies have shown, the learning of algebra represents a widespread problem, which must raise questions at all scholastic levels (Kieran, 1992, Bell, 1995). By its very nature, the main difficulties that the topic can cause for students are of a semiotic nature. A good understanding of the meaning of algebraic terms could in many cases allow students to resolve algebraic expressions without carrying out algorithmic procedures which often lead to mistakes. As Duval (1988) underlines, it is necessary to introduce the student to different representations of the concepts being studied. According to Duval, the use of different semiotic registers allows teachers to simultaneously demonstrate the conceptual meaning to the student and to avoid them confusing or mistakenly identifying this concept with a single representation. All this becomes more relevant in algebra, where symbols play a central role. In Italian didactic practice, this conceptual training often does not happen and algebra learning is reduced to mere mechanical procedures which often cause the meaning of the algebraic term to be lost, always increasing the distance between personal and cultural meanings of the algebraic terms. We embrace the belief of Arcavi (1995) who claims it is certainly not by performing numerous equations that students are guided into algebraic reasoning. The difficulties highlighted and widely studied in the literature (Duval 2008; Radford, 2010), are studied and quantified at nationwide in the large-scale assessment test in this paper. Analysing test answers from a significant sample of students not only allowed the identification of the phenomenon in question, but also enabled the investigation and study of specific characteristics. The percentage numbers of the individual options supplied precise information about the mistakes made by students while analysis of the characteristic curve (Rasch, 1960) highlighted specific behaviour according to the ability level of students as identified in the test.

As already highlighted in earlier studies, data collection and analysis of the results of large-scale national and international assessment tests (see e.g. Ferretti, Giberti, Lemmo, 2018) can provide an opportunity to examine results through a very powerful quantitative lens and suggest (as in our case) specific issues about 
the macro-phenomena which emerge at a systematic level. In fact, these tests often reveal "phenomena" of students' behaviour when faced with various mathematics tasks and ultimately about their learning characteristics and processes, particularly regarding the dynamics and some causes of their difficulties. Statistical measurement and data analysis provide information not only regarding the global achievements at school system level, but also concerning typical student difficulties.

This research study suggests starting points for further research where qualitative and quantitative analysis performed on large-scale assessment tests may provide important elements for the study and future development of key constructs in mathematics education.

\section{Disclosure statement}

No potential conflict of interest was reported by the authors.

\section{Notes on contributors}

Federica Ferretti - Faculty of Education, Free University of Bozen-Bolzano, Italy.

\section{REFERENCES}

Arcavi, A. (1995). Teaching and Learning Algebra: Past, Present, and Future. Journal of Mathematical Behavior, 14(1), 145-62. https://doi.org/10.1016/0732-3123(95)90033-0

Bell, A. (1995). Purpose in school algebra. The Journal of Mathematical Behavior, 14(1), 41-73. https://doi.org/10.1016/0732-3123(95)90023-3

Bolondi, G., Ferretti, F., \& Giberti, C. (2018). Didactic Contract as a Key to Interpreting Gender Differences in Maths. Journal of Educational, Cultural and Psychological Studies (ECPS Journal), (18), 415-435. https://doi.org/10.7358/ecps-2018-018-bolo

Callingham, R., \& Bond, T. (2006). Research in mathematics education and Rasch measurement. Mathematics Education Research Journal, 18(2), 1-10. https://doi.org/10.1007/BF03217432

De Lange, J. (2007). Large-Scale Assessment and Mathematics Education. In Frank K. Lester, Jr. (Ed.), Second Handbook of Research on Mathematics Teaching and Learning (pp. 1111-1142). Charlotte, NC: National Council of Teachers of Mathematics (NCTM).

Duval, R. (1988). Pour une approche cognitive des problèmes de géométrie en termes de congruences. Annales de Didactique et de Sciences Cognitives, 1, 57-74. Strasbourg, France: IREM et Université Louis Pasteur.

Duval, R. (1993). Registres de représentations sémiotique et fonctionnement cognitif de la pensée. Annales de Didactique et de Sciences Cognitives, 5, 37-65. Strasbourg, France: IREM et Université Louis Pasteur.

Duval, R. (1995). Sémiosis et pensée humaine: Registres sémiotiques et apprentissages intellectuels. Berne: Peter Lang.

Duval, R. (2008). Eight problems for a semiotic approach in mathematics education. In L. Radford, G. Schubring, \& F. Seeger (Eds.), Semiotics in mathematics education: Epistemology, history, classroom, and culture (pp. 39-61). Rotterdam: Sense Publishers.

Ernest, P. (1991). The philosophy of mathematics education. London, Falmer Press.

Ferretti, F., Giberti, C., \& Lemmo, A. (2018). The Didactic Contract to interpret some statistical evidence in mathematics standardized assessment tests. EURASIA Journal of Mathematics, Science and Technology Education, 14(7), 2895-2906. https://doi.org/10.29333/ejmste/90988

Ferretti, F., Lemmo, A. \& Martignone, F. (2018). Attained curriculum and external assessment in Italy: how to reflect on them?. In Y. Shimizu and R. Vithal (Eds.), Proceedings of the Twenty-fourth ICMI Study School Mathematics Curriculum Reforms: Challenges, Changes and Opportunities (pp.381-388). Tsukuba, Japan: ICMI.

Gestinv 2.0. Interactive archive of the INVALSI tests. http://www.gestinv.it (ver. 15.04.2019).

Godino J., Batanero C. (1994). Significado institucional y personal de los objectos matematicos. Recherches en Didactique des Mathématiques. 14(3), 325-355.

Johnson, R. B., \& Onwuegbuzie, A. J. (2004). Mixed methods research: A research paradigm whose time has come. Educational Researcher, 33(7), 14-26. https://doi.org/10.3102/0013189X033007014 
Hart, L.C., Smith, S.Z., Swars, S. L., Smith, M.E. (2009). An Examination of Research Methods in Mathematics Education (1995-2005). Journal of Mixed Methods Research 3(1), 26-41. https://doi.org/10.1177/1558689808325771

Kieran, C. (1992). The learning and teaching of school algebra. In D. Grouws (ed.), Handbook of research on mathematics teaching and learning. Macmillan, New York.

Kieran, C. (1995). A new look at school algebra-Past, present, and future. The Journal of Mathematical Behavior, 14(1), 7-12. https://doi.org/10.1016/0732-3123(95)90020-9

Kieran, C. (2007). Learning and teaching algebra at the middle school through college levels: Building meaning for symbols and their manipulation. Second handbook of research on mathematics teaching and learning, 2, 707-762.

Kieran, C. (2014). Algebra teaching and learning. Encyclopedia of Mathematics Education, 27-32. https://doi.org/10.1007/978-94-007-4978-8_6

Radford, L. (2002). The seen, the spoken and the written: A semiotic approach to the problem of objectification of mathematical knowledge. For the learning of mathematics, 22(2), 14-23.

Radford, L. (2008). The ethics of being and knowing: Towards a cultural theory of learning. In Semiotics in mathematics education (pp. 215-234). Rotterdam: Sense Publishers

Radford, L. (2010). Algebraic thinking from a cultural semiotic perspective. Research in Mathematics Education, 12(1), 1-19. https://doi.org/10.1080/14794800903569741

Rasch, G. (1960). Probabilistic models for some intelligence and attainment tests. Copenhagen: Denmarks Paedagogiske Institut.

Sfard, A. (1995). The development of algebra: Confronting historical and psychological perspectives. The Journal of Mathematical Behavior, 14(1), 15-3. https://doi.org/10.1016/0732-3123(95)90022-5 